\title{
Comment on "Application of Fresnel diffraction to nondestructive measurement of the refractive index of optical fibers"
}

\author{
Soumia I. Taleb, Cristian Neipp,* and Jorge Francés \\ University of Alicante, Department of Physics, Systems Engineering, and Signal Theory, \\ Alicante, Spain
}

\begin{abstract}
We examine the validity of Eq. (5) in [A. Sabatyan and M. T. Tavassoly, "Application of Fresnel diffraction to nondestructive measurement of the refractive index of optical fibers," Opt. Eng., 2007, Vol. 46(12), pp. 128001-1-128001-7] to describe the diffraction pattern of an optical fiber. We show that Eq. (5) must be changed to account for the phase introduced by the fiber correctly. In particular, we show that, to agree with the implicit criterion for representing traveling waves, given when using the Fresnel-Kirchoff integral [Eq. (4)], the phase introduced by the object must be carried by positive exponentials. The results obtained correcting Eq. (5) are compared with those obtained by the rigorous solution of Maxwell equations in the optical fiber. We demonstrate the importance of adequately taking into account the criterion implicitly assumed in the Fresnel-Kirchoff integral to represent traveling waves. (C) 2020 Society of PhotoOptical Instrumentation Engineers (SPIE) [DOI: 10.1117/1.OE.59.6.060401]
\end{abstract}

Keywords: Fresnel diffraction; optical fiber; phase object; metrology.

Paper 20200341C received Apr. 4, 2020; accepted for publication Jun. 4, 2020; published online Jun. 18, 2020.

\section{Introduction}

A. Sabatyan and M. T. Tavassoly proposed a nondestructive technique, based on Fresnel diffraction from phase objects, for specifying the refractive indices of optical fibers without requiring an index matching liquid. The method proposed is interesting and accurate, although we suggest two changes in Eq. (5) to predict the diffraction pattern of an optical fiber correctly.

First, we found one erratum; the authors define constant $K^{\prime}$ as $K^{\prime}=K \exp \left(i z^{\prime}\right) / \sqrt{z^{\prime}}$, but it must be

$$
K^{\prime}=K \exp \left(i k z^{\prime}\right) / \sqrt{\lambda z^{\prime}} .
$$

On the other hand, a more important aspect is related to the way the phase of the fiber is computed. As can be seen in Eq. (5), the phase introduced by the fiber and the surroundings is carried by negative exponentials. For instance, if $\varphi_{\mathrm{Cl}}$ stands for the phase introduced by the cladding, its effect is included in Eq. 5) by $\exp \left(-i \varphi_{\mathrm{Cl}}\right)$. The use of this criterion means that for a wave traveling from left to right, the phase becomes more negative. Nonetheless, if the Fresnel-Kirchoff integral is used as it stands in Eq. (4) of Ref. 1, the positive exponentials $\exp (i k r)$ represent diverging spherical waves traveling from one point of the input plane to another of the output plane (from left to right). Therefore, two different criteria are used to represent waves traveling in space. A good discussion of positive versus negative phases can be found in Ref. 2. One can choose one or the other criteria, but not both at the same time. What we want to stress in this comment is that using the Fresnel-Kirchoff integral to simulate the diffraction of an object implicitly selects a criterion that must be kept in all related calculations.

In this particular case, Eq. (5) gives the correct results if the negative exponentials are changed to positive exponentials. That is, Eq. (5) must read as

*Address all correspondence to Cristian Neipp, E-mail: cristian@ua.es

0091-3286/2020/\$28.00 ㄷ 2020 SPIE 


$$
\begin{aligned}
U(Q)= & \frac{K^{\prime \exp \left(i \varphi_{S u}\right)}}{B}\{1+C(\alpha)-C(\beta)+i[1+S(\alpha)-S(\beta)]+ \\
& +B\left[\int_{-b}^{-a} \exp \left[i \varphi_{\mathrm{Cl}}\left(x^{\prime}\right)\right] \exp \left[i k \frac{\left(x-x^{\prime}\right)^{2}}{2 z^{\prime}}\right] \mathrm{d} x^{\prime}\right. \\
& +\int_{-a}^{a} \exp \left\{i\left[\varphi_{\mathrm{Cl}}\left(x^{\prime}\right)++\varphi_{\mathrm{Co}}\left(x^{\prime}\right)\right]\right\} \exp \left[i k \frac{\left(x-x^{\prime}\right)^{2}}{2 z^{\prime}}\right] \mathrm{d} x^{\prime} \\
& \left.\left.+\int_{a}^{b} \exp \left[i \varphi_{\mathrm{Cl}}\left(x^{\prime}\right)\right] \exp \left[i k \frac{\left(x-x^{\prime}\right)^{2}}{2 z^{\prime}}\right] \mathrm{d} x^{\prime}\right]\right\},
\end{aligned}
$$

where $K^{\prime}$ defined as Eq. (1) in this comment.

To demonstrate this, we compare the results obtained using the Fresnel-Kirchoff integral with those obtained rigorously solving Maxwell equations for TE polarization for an optical fiber. We calculate the scattered field by a dielectric cylinder composed of two layers when a plane wave is incident onto the cylinder using the Rigorous theory developed in two references. ${ }^{3,4}$

\section{Results and Discussion}

In this section, we compare the results obtained using the Fresnel-Kirchoff equation with Fresnel approximation and those obtained rigorously solving Maxwell equations to calculate the diffraction pattern of an optical fiber that has internal radii $a=25.5 \mu \mathrm{m}, b=62.5 \mu \mathrm{m}$ and refractive indices of the cladding and the core $n_{\mathrm{Cl}}=1.458$ and $n_{\mathrm{Co}}=1.475$, respectively. The wavelength of the incident light was assumed to be $\lambda=633 \mathrm{~nm}$ in all cases.

In Fig. 1, the intensity of the diffraction pattern created by the optical fiber is represented as a function of the axial distance. In this case, Eq. (5) of Ref. 1 (expressing the phase introduced by the fiber with negative exponentials) was used for the simulations of the Fresnel pattern. The results are compared with those obtained by the rigorous solution of Maxwell equations. The discrepancies between both methods are obvious, and the two patterns seem to be displaced by a phase difference of $\pi$.

In Fig. 2, the intensity of the diffraction pattern created by the optical fiber is represented as a function of the axial distance, but in this case, Eq. (2) of this comment was used for

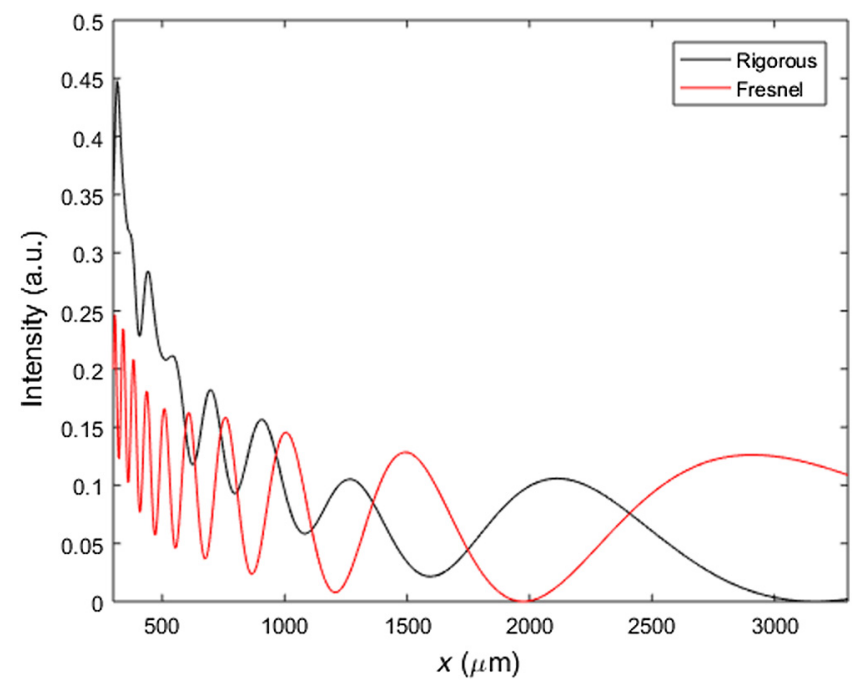

Fig. 1 Intensity of the diffraction pattern as a function of the axial distance $z$ for an optical fiber with internal radii $a=25.5 \mu \mathrm{m}, b=62.5 \mu \mathrm{m}$, and refractive indices $n_{\mathrm{Cl}}=1.458$ and $n_{\mathrm{Co}}=1.475$. The simulation made using the Fresnel-Kirchoff equation was obtained assuming negative exponentials to account for the phase introduced by the optical fiber [Eq. (5) of Ref. 1]. 


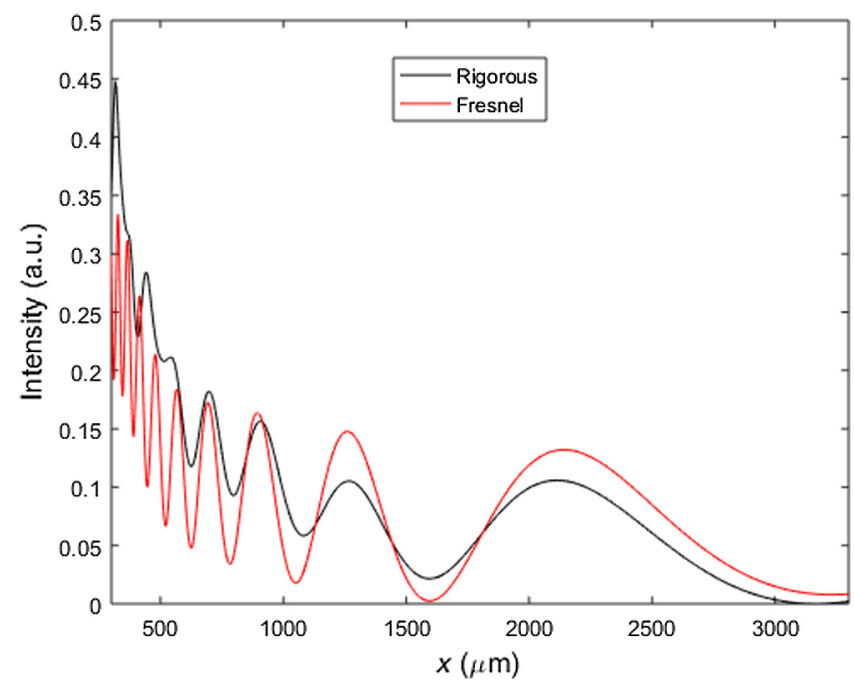

Fig. 2 Intensity of the diffraction pattern as a function of the axial distance $z$ for an optical fiber with internal radii $a=25.5 \mu \mathrm{m}, b=62.5 \mu \mathrm{m}$, and refractive indices $n_{\mathrm{Cl}}=1.458$ and $n_{\mathrm{Co}}=1.475$. The simulation made using the Fresnel-Kirchoff equation was obtained assuming positive exponentials to account for the phase introduced by the optical fiber [Eq. (2) of this comment].

the simulations of the Fresnel pattern. It can be seen that the overall behavior of both patterns are similar, with increasing agreement as the axial distance grows.

In Fig. 3, the intensity of the diffraction pattern created by the optical fiber is represented as a function of the distance to the center of the diffraction pattern. The diffraction pattern was calculated at an axial distance of $23 \times 10^{3} \mu \mathrm{m}$ from the fiber. Equation (5) of Ref. 1 was used for the simulations of the Fresnel pattern. It can be seen that both methods yield different results. If the phase difference carried by the incident wave after passing through the fiber is introduced in Eq. (5) of Ref. 1 with positive exponentials [Eq. (2)] both models predict the same behavior, which can be clearly observed in Fig. 4.

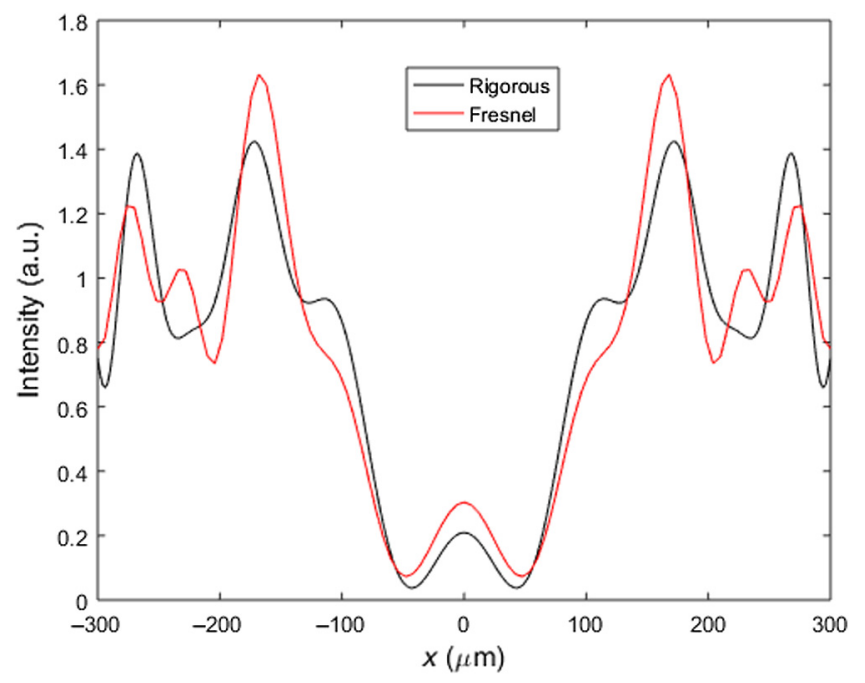

Fig. 3 Intensity of the diffraction pattern as a function of the distance to the center of the diffraction pattern for an optical fiber with internal radii $a=25.5 \mu \mathrm{m}, b=62.5 \mu \mathrm{m}$, and refractive indices $n_{\mathrm{Cl}}=1.458$ and $n_{\mathrm{Co}}=1.475$. The intensities were calculated assuming that the diffraction pattern is measured at an axial distance $z=23 \times 10^{3} \mu \mathrm{m}$ from the fiber. The simulation made using the Fresnel-Kirchoff equation was obtained assuming negative exponentials to account for the phase introduced by the optical fiber [Eq. (5) of Ref. 1]. 


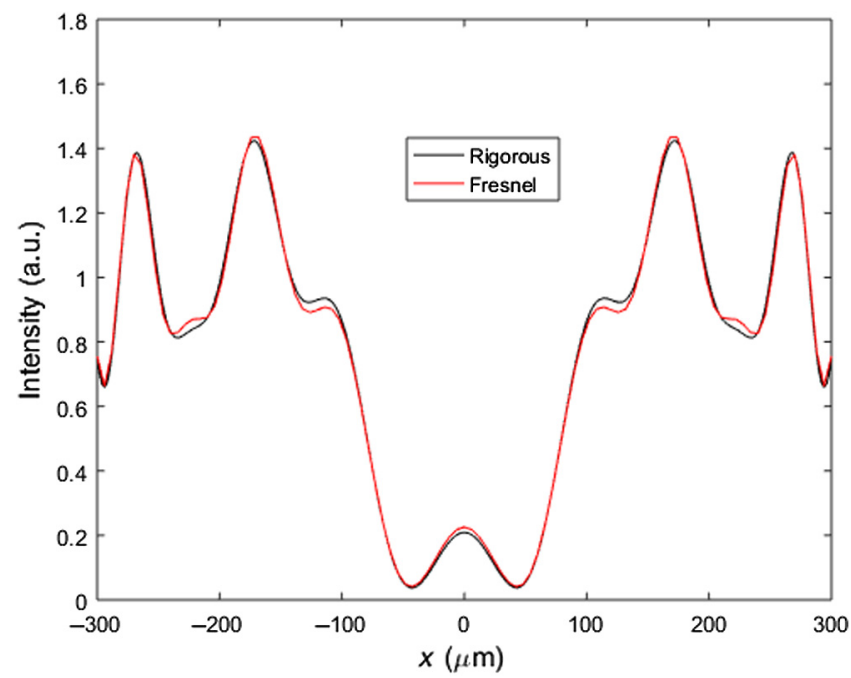

Fig. 4 Intensity of the diffraction pattern as a function of the distance to the center of the diffraction pattern for an optical fiber with internal radii $a=25.5 \mu \mathrm{m}, b=62.5 \mu \mathrm{m}$, and refractive indices $n_{\mathrm{Cl}}=1.458$ and $n_{\mathrm{Co}}=1.475$. The intensities were calculated assuming that the diffraction pattern is measured at an axial distance $z=23 \times 10^{3} \mu \mathrm{m}$ from the fiber. The simulation made using the Fresnel-Kirchoff equation was obtained assuming positive exponentials to account for the phase introduced by the optical fiber [Eq. (2) of this comment].

Finally, we want to comment that the fits to the experimental data made with the function of Eq. (2) of this comment instead of Eq. (5) of Sabatyan et al. only slightly change the fitted parameters. For instance, for the fitting of Fig. 6(a), we obtained the following parameters: radii $a=25.1 \mu \mathrm{m}, b=62.5 \mu \mathrm{m}$, and refractive indices $n_{\mathrm{Cl}}=1.457$ and $n_{\mathrm{Co}}=1.472$. Figure 5 shows the theoretical functions obtained with the two different expressions used in both articles. The values of Sabatyan et al. correspond to the theoretical curve of Fig. 6(a), with the fitted parameters: $a=25.5 \mu \mathrm{m}, b=62.5 \mu \mathrm{m}$, and refractive indices $n_{\mathrm{Cl}}=1.458$ and $n_{\mathrm{Co}}=1.4747$.

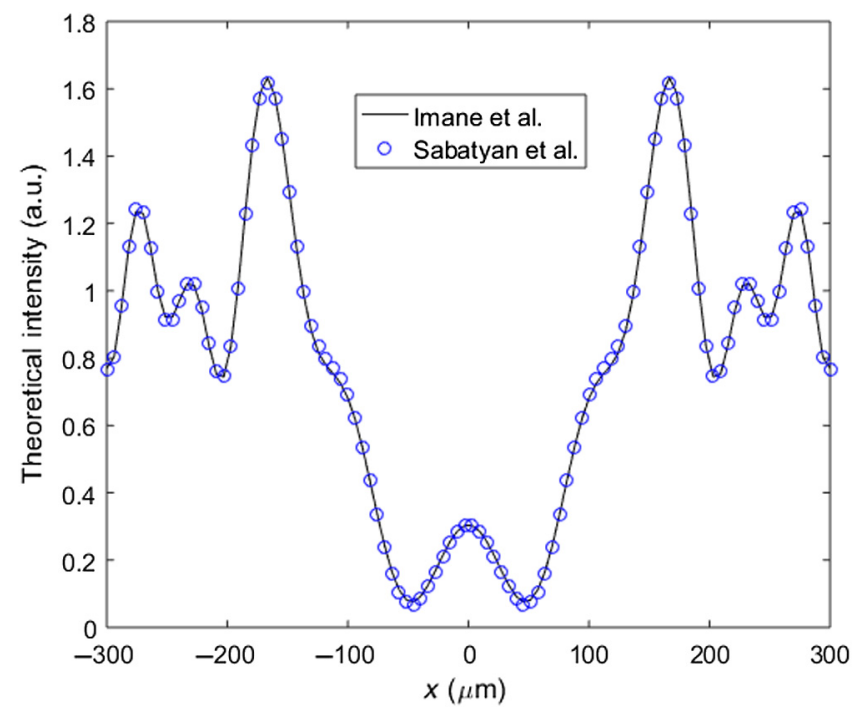

Fig. 5 Intensity of the diffraction pattern as a function of the distance to the center of the diffraction pattern for an optical fiber. The intensities were calculated assuming that the diffraction pattern is measured at an axial distance $z=23 \times 10^{3} \mu \mathrm{m}$ from the fiber. The dots correspond to the theoretical function of Eq. (5) of Ref. 1 with internal radii $a=25.5 \mu \mathrm{m}, b=62.5 \mu \mathrm{m}$, and refractive indices $n_{\mathrm{Cl}}=1.458$ and $n_{\mathrm{Co}}=1.4747$. The solid line corresponds to Eq. (2) of this comment with internal radii $a=25.1 \mu \mathrm{m}, b=62.5 \mu \mathrm{m}$, and refractive indices $n_{\mathrm{Cl}}=1.457$ and $n_{\mathrm{Co}}=1.472$. 


\section{Conclusions}

It has been demonstrated that the when using the Fresnel-Kirchoff integral to calculate the diffraction pattern of a phase object, using either positive or negative exponentials to carry the phase introduced by the object affect the final results. Therefore, the same criterion as that implicitly assumed in the proper Fresnel-Kirchoff integral must be chosen.

\section{Acknowledgments}

This work was supported by the "Ministerio de Economía Industria y Competitividad" (Spain) under Project No. FIS2017-82919-R (MINECO/AEI/FEDER, UE).

\section{References}

1. A. Sabatyan and M. T. Tavassoly, "Application of Fresnel diffraction to nondestructive measurement of the refractive index of optical fibers," Opt. Eng. 46(12), 128001 (2007).

2. J. W. Goodman, Introduction to Fourier Optics, McGraw-Hill, New York (1996).

3. H. Wang et al., "Theory of enhanced Raman scattering for circular stratified semiconductor cylinder," J. Phys.: Condens. Matter. 19, 246222 (2007).

4. M. Kerker and E. Matijevic, "Scattering of electromagnetic waves from concentric infinite cylinders," J. Opt. Soc. Am. 51, 506-508 (1961).

Soumia I. Taleb received his master's degree in photonics with a specialty in optical telecommunications from Abou Bekr Belkaid University, Algeria, in 2013. Currently, she is enrolled in the doctoral program. She has been a researcher and student at the Diffractive Optical Group Research of the University Institute of Applied Physics in Science and Technology at the University of Alicante since 2017. Her research interests are volume holography and numerical simulation.

Cristian Neipp received his degree in physical sciences from the University of Salamanca and his $\mathrm{PhD}$ from the University of Alicante. He currently holds the position of professor at the same university and has been exercising his teaching work at the Polytechnic School of the University of Alicante since 1998. He has co-authored more than 120 research articles in the areas of optics, applied physics, and mechanical engineering.

Jorge Francés received his BE degree in telecommunication engineering from the Polytechnic University of Valencia, Valencia, Spain, in 2007 and his PhD from the University of Alicante in 2011. From 2008 to 2018, he worked as an assistant lecturer at the University of Alicante. Since 2018, he has been working as a senior lecturer. His main research interests include physical optics, sound and vibration, and numerical simulation. 